\title{
Frequency of Mucositis Among Head and Neck Cancer Patients Receiving Three-Dimensional (3D) Radiotherapy
}

Talha Laique ${ }^{1}$, Ambreen Shabbir ${ }^{2}$, Muhammad Ahmad Bilal Ahmadani ${ }^{3}$, Maher Sohail Yaseen ${ }^{4}$, Ayesha Ahmad $^{5}$, Jahanzeb Malik ${ }^{6}$

1. Pharmacology, Lahore Medical and Dental College, Lahore, PAK 2. Pathology, Combined Military Hospital (CMH) Institute of Medical Sciences, Multan, PAK 3. Radiology, Cardiac Centre, Bahawalpur, PAK 4. Physiology, D.G Khan Medical College, Dera Ghazi Khan, PAK 5. Radiation Oncology, Institute of Nuclear Medicine and Oncology (INMOL), Lahore, PAK 6. Cardiology, Rawalpindi Institute of Cardiology, Rawalpindi, PAK

Corresponding author: Talha Laique, talhalaique51@gmail.com

\section{Abstract}

\section{Background and objective}

Head and neck cancer can arise from any site like hypo-pharynx, oro-pharynx, lip, oral cavity, or larynx. Almost 90\% of them are head and neck squamous cell carcinomas (HNSCC). In the current project, the goal was to determine the frequency of acute side effects in terms of mucositis during and immediately postirradiation period in patients receiving concurrent three-dimensional (3D) radiotherapy in a tertiary care hospital.

\section{Methodology}

This descriptive case series with 106 enrolled patients was carried out from December 2019 to May 2020 at the Department of Radiation Oncology following approval. All patients were given radiotherapy or chemoradio-therapy as per the clinician's advice and hospital protocol. All patients were evaluated at pre radiation time, at weekly intervals during treatment and at 11 weeks from 1st radiation fraction. Data was entered and analyzed by Statistical Package for Social Sciences, version 20 (SPSS Inc., Chicago, IL). Chi-square and Fisher's exact test was applied as $\mathrm{p}$-value $\leqslant 0.05$ was considered significant.

\section{Results}

In the present study, all patients ( $\mathrm{n}=106)$ showed a mean age of $57.8 \pm 8.3$ years. There was a gradual increase in grades of mucositis in all patients after treatments until seven weeks. After one month of post-treatment, a decrease in grades of mucositis was observed in all patients.

Received 06/29/2020 Review began 07/02/2020 Review ended 07/03/2020 Published 07/09/2020

() Copyright 2020 Laique et al. This is an open access article distributed under the terms of the Creative Commons Attribution License CC-BY 4.0., which permits unrestricted use, distribution, and reproduction in any medium, provided the original author and source are credited.

\section{Conclusion}

Acute side effects appeared in all patients receiving 3D radiation therapy (RT) although the treatment response was good. Hence, we concluded it has a high incidence of treatment-related toxicities but it is safe.

Categories: Radiation Oncology, Radiology

Keywords: 3-d radiotherapy, mucositis

\section{Introduction}

Head and neck cancer is a heterogeneous disease that can arise from any site like hypo-pharynx, oropharynx, lip, oral cavity, nasopharynx, or larynx [1]. Almost 90\% of them are head and neck squamous cell carcinomas (HNSCC). It is ranked sixth among all cancers globally with reported new patients $(630,000)$ identified per year. Globally, $12 \%$ of all malignancies are constituted by oral and head and neck cancer (OHNC). Oral $(400,000)$ and laryngeal $(160,000)$ cancer cases usually develop each year according to previous estimation [2]. Head and neck cancers account for approximately 45\%-55\% survival rate for patients having it after the five years of diagnosis and treatment [3]. Males (21\%) suffer more from head and neck cancers than females (11\%) across the Pakistani population respectively [4,5].

Head and neck cancers are mainly due to various factors including demographic parameters (smoking, alcohol intake), eating patterns, infections with viruses, and family history [6]. Concurrent radiation therapy (RT) with cisplatin has improved locoregional control in squamous cell cancer. It is now the basic component of a multi-disciplinary approach for head and neck cancer treatment. The purpose of RT is to damage tumor maximally with minimum side effects using modalities like two-dimensional (2D)/three-dimensional (3D)/intensity-modulated radiation therapy (IMRT) though it is linked with several short-term and longterm side effects [7-9]. 


\section{Cureus}

An adverse event (AE) is any unwanted effect produced as a result of a medical treatment or procedure. Toxicity criteria of the Radiation Therapy Oncology Group (RTOG) and the European Organization for Research and Treatment of Cancer (EORTC) defined acute side effects like mucositis, dermatitis, and dry mouth, etc into grades to show organ toxicity among subjects receiving RT [7].

Regarding the high incidence of head and neck cancers among our population with limited data available regarding acute side effects of RT, we planned the current study to see the frequency of acute side effects (mucositis) associated with 3D-radiotherapy technique (3D-RT) in head and neck cancers treatment. It helped us to observe how frequently mucositis developed among patients on RT for continuous 11 weeks.

\section{Materials And Methods}

This descriptive case series with 106 enrolled patients was carried out from December 2019 to May 2020 at the Department of Radiation Oncology, Institute of Nuclear Medicine and Oncology (INMOL), Lahore, following the approval by the Hospital's Ethical Committee. The Sample size (106) cases were calculated with a 95\% confidence level, 9\% margin of error, and taking an expected percentage of mucositis with 3D-RT as $33.3 \%$ (9). Patients were enrolled by non-probability consecutive sampling. All patients of head and neck cancer including both genders with a mean age of $57.8 \pm 8.3$ years presenting to the radio-oncology department were enrolled. Exclusion criteria involved patients who were unable to give informed consent, pre-existing skin diseases, any second malignancy or metastasis, and pregnant. Written informed consent was taken from the patient at the time of enrollment.

Data was entered and analyzed by Statistical Package for Social Sciences, version 20 (SPSS Inc., Chicago, IL). Quantitative data like age (in years) and total radiation dose were presented as Mean \pm SD. The categorical data like gender, site of cancer, and acute side effects were offered as frequency and percentages. Chi-square and Fisher's exact test was used to compare the frequency of acute side effects among different based on gender and the site of cancer with $p$-value $\leqslant 0.05$ was considered significant.

\section{Results}

Among 106 enrolled patients, age ranged from 25-70 years. Demographic parameters like age and dose of radiation as mean \pm SD are shown in Table 1 below. All these parameters were noted at the time of enrollment.

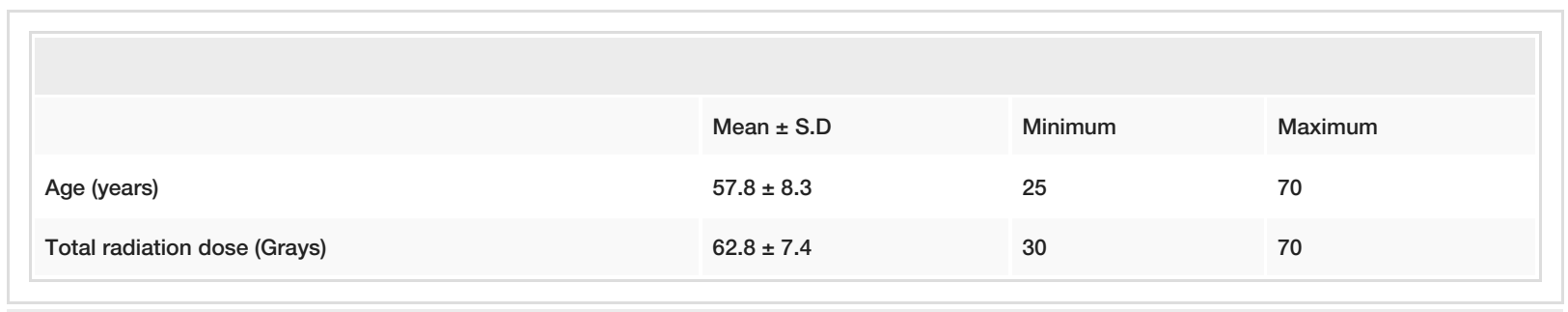

TABLE 1: Descriptive statistics of patients with respect to age and total radiation dose

The distribution of patients enrolled in the study concerning gender and sites of cancer involved is shown in Table 2 below. 


\section{Cureus}

Table 2: Distribution of participants with respect to gender and site of cancer

\begin{tabular}{|llll} 
Variable & Category & Frequency & Percentage (\%) \\
Male & Fender & 44 & 41.5 \\
& Fharynx & 62 & 58.5 \\
& Hypopharynx & 23 & 21.7 \\
Site & Larynx & 22 & 20.8 \\
& Nasopharynx & 8 & 7.5 \\
& Oral cavity & 22 & 20.8
\end{tabular}

TABLE 2: Distribution of participants with respect to gender and sites of cancer

There was a gradual increase in grades of mucositis in all patients after treatments with 3D-RT until seven weeks. After one month of post-treatment, a decrease in grades of mucositis in all patients was observed. The result regarding distribution according to grades of mucositis among enrolled patients are summarized in Table 3 .

\begin{tabular}{|c|c|c|c|c|c|c|}
\hline Week & Grade 0 & Grade 1 & Grade 2 & Grade 3 & Grade 4 & Grade 5 \\
\hline 1st & 79 (74.5\%) & 27 (25.5\%) & $0(0 \%)$ & $0(0 \%)$ & $0(0 \%)$ & $0(0 \%)$ \\
\hline $2^{\text {nd }}$ & 64(60.4\%) & 42 (39.6\%) & $0(0 \%)$ & $0(0 \%)$ & $0(0 \%)$ & $0(0 \%)$ \\
\hline $3^{\text {rd }}$ & $1(0.9 \%)$ & 79 (74.5\%) & $26(24.5 \%)$ & $0(0 \%)$ & $0(0 \%)$ & $0(0 \%)$ \\
\hline $4^{\text {th }}$ & $0(0 \%)$ & $78(73.6 \%)$ & $28(26.4)$ & $0(0 \%)$ & $0(0 \%)$ & $0(0 \%)$ \\
\hline $5^{\text {th }}$ & $0(0 \%)$ & $38(35.8 \%)$ & $67(63.2 \%)$ & $1(0.9 \%)$ & $0(0 \%)$ & $0(0 \%)$ \\
\hline $6^{\text {th }}$ & $0(0 \%)$ & $0(0 \%)$ & 79 (74.5\%) & 27 (25.5\%) & $0(0 \%)$ & $0(0 \%)$ \\
\hline $7^{\text {th }}$ & $0(0 \%)$ & $0(0 \%)$ & 52 (49.1\%) & $49(46.2 \%)$ & $5(4.7 \%)$ & $0(0 \%)$ \\
\hline $11^{\text {th }}$ & 56 (52.8\%) & $49(46.2 \%)$ & $1(0.9 \%)$ & $0(0 \%)$ & $0(0 \%)$ & $0(0 \%)$ \\
\hline
\end{tabular}

TABLE 3: Showing distribution of patients according to the grade of mucositis

Chi-square and Fisher's exact test was used to compare the frequency of mucositis grades between male and female patients (Table 4). The grades of mucositis in male patients versus female patients was significantly different on the 4 th week of treatment alone. It means that mucositis of varying grades developed among patients at the 4 th week of treatment more than any other week till the 7th week of treatment. 


\section{Cureus}

Table 4: Showing comparison of grade of Mucositis between both genders

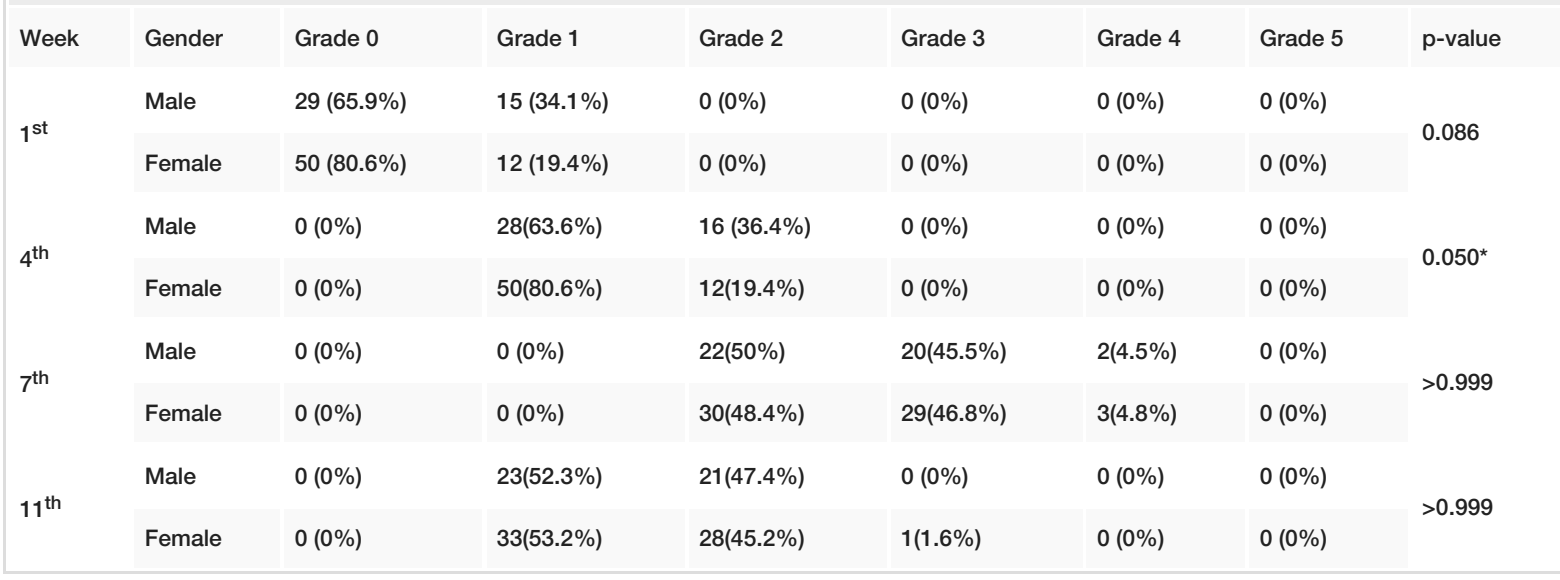

TABLE 4: Showing comparison of the grade of mucositis between both genders

Chi-square and Fisher's exact test was used to compare the frequency of mucositis grades among sites at 1st, 4th and 7th week of treatment as shown in Table 5. This was done in order to observe the development of mucositis at start after the 1st week of treatment and at end of treatment at the 7th week involving various sites in head and neck region. In the current study, we observed it one month post treatment at the 11th week to see improvement in its grades. There was a significant difference in the frequency of mucositis grades among sites involved in the 7th week of treatment. This showed grade 2 and 3 mucositis developed at all sites of head and neck region.

\begin{tabular}{|c|c|c|c|c|c|c|c|c|}
\hline Week & Site & Grade 0 & Grade 1 & Grade 2 & Grade 3 & Grade 4 & Grade 5 & $p$-value \\
\hline \multirow{4}{*}{$1^{\text {st }}$} & Pharynx & 14 (60.9\%) & $9(39.1 \%)$ & $0(0 \%)$ & $0(0 \%)$ & $0(0 \%)$ & $0(0 \%)$ & \multirow{4}{*}{0.321} \\
\hline & Hypopharnx & $15(68.2 \%)$ & $7(31.8 \%)$ & $0(0 \%)$ & $0(0 \%)$ & $0(0 \%)$ & $0(0 \%)$ & \\
\hline & Nasopharyx & 17 (77.3\%) & $5(22.7 \%)$ & $0(0 \%)$ & $0(0 \%)$ & $0(0 \%)$ & $0(0 \%)$ & \\
\hline & Oral cavity & 26 (83.9\%) & $5(16.1 \%)$ & $0(0 \%)$ & $0(0 \%)$ & $0(0 \%)$ & $0(0 \%)$ & \\
\hline \multirow{4}{*}{$7^{\text {th }}$} & Pharynx & $0(0 \%)$ & $0(0 \%)$ & $6(26.1 \%)$ & $14(60.9 \%)$ & $3(13.0 \%)$ & $0(0 \%)$ & \multirow{4}{*}{$0.012^{*}$} \\
\hline & Hypopharnx & $0(0 \%)$ & $0(0 \%)$ & 13 (59.1\%) & $8(36.4 \%)$ & $1(4.5 \%)$ & $0(0 \%)$ & \\
\hline & Nasopharyx & $0(0 \%)$ & $0(0 \%)$ & $12(54.5 \%)$ & 10 (45.5\%) & $0(0 \%)$ & $0(0 \%)$ & \\
\hline & Oral cavity & $0(0 \%)$ & $0(0 \%)$ & 20 (64.5\%) & 11 (35.5\%) & $0(0 \%)$ & $0(0 \%)$ & \\
\hline \multirow{4}{*}{$11^{\text {th }}$} & Pharynx & $0(0 \%)$ & $7(30.4 \%)$ & 15 (65.2\%) & $1(4.3 \%)$ & $0(0 \%)$ & $0(0 \%)$ & \multirow{4}{*}{0.104} \\
\hline & Hypopharynx & $0(0 \%)$ & 13(59.1\%) & $9(40.9 \%)$ & $0(0 \%)$ & $0(0 \%)$ & $0(0 \%)$ & \\
\hline & Nasopharyx & $0(0 \%)$ & $12(54.5 \%)$ & 10 (45.5\%) & $0(0 \%)$ & $0(0 \%)$ & $0(0 \%)$ & \\
\hline & Oral cavity & $0(0 \%)$ & $21(67.7 \%)$ & 10 (32.3\%) & $0(0 \%)$ & $0(0 \%)$ & $0(0 \%)$ & \\
\hline
\end{tabular}

TABLE 5: Showing comparison of the grades of mucositis among sites involved

\section{Discussion}

RT was planned for patients with HNSCC. It was done to determine the frequency of acute side effects in terms of mucositis during and immediately post-irradiation period (upto 11 weeks from the first fraction) in patients receiving concurrent 3D-RT. Thus we examined the efficacy and safety of RT in non-metastatic, stage I to stage III HNSCC patients, very representative of our daily practice in our country Pakistan [10]. There was a significant clinical response to RT in our patients. No death was reported in our groups. Acute 
side effects related to RT are not reviewed routinely in our setups. Pakistan is one of the high burden countries for head and neck cancers.

Our enrolled sample size was 106 in the current project. The number of patients conformed with one another previous study who in their project enrolled 221 patients to determine the presenting symptoms in head and neck cancer patients [11]. In contrast, one study carried on the Brazilian population included 30 head and neck cancer patients [10].

Age (mean \pm SD) of enrolled patients in our study was $57.8 \pm 8.3$ years in conformity with the previous study where age (mean \pm SD) of enrolled head and neck cancer patients among European population was $64 \pm 12$ years (Table 1). In past researches, the median age was 53 (37-68) rather than mean of age [12].

In the current study, tumors involving various head and neck sites like pharynx, hypopharynx, larynx, larynx, and oral cavity were considered. The most common site was oral cavity 31 (29.2\%) and the least common site was larynx 8 (7.5\%) as given in Table 2 . Our results were in line with previous studies [10].

In our project, acute side effects like mucositis were seen during and immediately after post-treatment at four weeks interval. There was a gradual increase in grades of mucositis in all patients after treatments until seven weeks. Mucositis of grade 1 was observed in only 27 (25.5\%) patients after one week of treatment. At the 4 th week after treatment, mucositis of grade 1 and 2 was observed in 106 (100.0\%) patients. At the 7th week of treatment, mucositis of grade 4 was observed in five (4.7\%) patients, mucositis of grade 2 and 3 was observed in 52 (49.1\%) and 49 (46.2\%) patients, respectively. After one month of post-treatment, a decrease in grades of mucositis in all patients was observed as shown in Table 3. Our findings were in line with previous studies of head and neck cancer treatment with chemo-radiotherapy [13].

Our study had several limitations like financial constraints and fewer resources. We did not perform positron emission tomography (PET) scan and genetic study to see genetic variability among enrolled subjects.

\section{Conclusions}

Acute side effects appeared in all patients receiving 3D-RT although the treatment response was good. Hence, we concluded 3D-RT has a high incidence of treatment-related toxicities but is relatively safe. Mucositis of varying grades developed till the completion of therapy but improved later at one-month post treatment. It was a reversible therapy-related toxicity but not life threatening.

\section{Additional Information \\ Disclosures}

Human subjects: Consent was obtained by all participants in this study. INMOL, Lahore issued approval Nil. This descriptive case series with 106 enrolled patients was carried out from December 2019 to May 2020 at the Department of Radiation Oncology, INMOL, Lahore following the approval by the Hospital's Ethical Committee. Animal subjects: All authors have confirmed that this study did not involve animal subjects or tissue. Conflicts of interest: In compliance with the ICMJE uniform disclosure form, all authors declare the following: Payment/services info: All authors have declared that no financial support was received from any organization for the submitted work. Financial relationships: All authors have declared that they have no financial relationships at present or within the previous three years with any organizations that might have an interest in the submitted work. Other relationships: All authors have declared that there are no other relationships or activities that could appear to have influenced the submitted work.

\section{Acknowledgements}

I would like to acknowledge the hard work of Department of Radiation Oncology, INMOL, Lahore. I am thankful to Allah, all my colleagues and teachers for their help.

\section{References}

1. Razak AR, Siu LL, Liu FF, Ito E, O'Sullivan B, Chan K: Nasopharyngeal carcinoma: the next challenges . Eur J Cancer. 2010, 46:1967-78. 10.1016/j.ejca.2010.04.004

2. Gupta B, Johnson NW, Kumar N: Global epidemiology of head and neck cancers: a continuing challenge . Oncology. 2016, 91:13-23. 10.1159/000446117

3. Vigneswaran N, Williams MD: Epidemiologic trends in head and neck cancer and aids in diagnosis . Oral Maxillofac Surg Clin North Am. 2014, 26:123-41. 10.1016/j.coms.2014.01.001

4. Joshi P, Dutta S, Chaturvedi P, Nair S: Head and neck cancers in developing countries . Rambam Maimonides Med J. 2014, 5:e0009. 10.5041/RMMJ.10143

5. Herrero JI, Pardo F, D'avola D et.al: Risk factors of lung, head and neck, esophageal, and kidney and urinary tract carcinomas after liver transplantation: the effect of smoking withdrawal. Liver Transpl. 2011, 17:402-8. $10.1002 / 1 \mathrm{t} .22247$

6. Lambert R, Sauvaget C, de Camargo Cancela M, Sankaranarayanan R: Epidemiology of cancer from the oral cavity and oropharynx. Eur J Gastroenterol Hepatol. 2011, 23:633-41. 10.1097/MEG.0b013e3283484795 


\section{Cureus}

7. van der Veen ID, Nuyts S: Can intensity-modulated-radiotherapy reduce toxicity in head and neck squamous cell carcinoma. Cancers. 2017, 9:135. 10.3390/cancers9100135

8. Marur S, Forastiere AA: Head and neck cancer: changing epidemiology, diagnosis, and treatment. Mayo Clin Proc. 2008, 83:489-501. 10.4065/83.4.489

9. De Castro G Jr, Snitcovsky IM, Gebrim EM, Leitão GM, Nadalin W, Ferraz AR, Federico MH: High-dose cisplatin concurrent to conventionally delivered radiotherapy is associated with unacceptable toxicity in unresectable, non-metastatic stage IV head and neck squamous cell carcinoma. Eur Arch Otorhinolaryngol. 2007, 264:1475. 10.1007/s00405-007-0395-9

10. Alho OP, Teppo H, Mäntyselkä P, Kantola S: Head and neck cancer in primary care: presenting symptoms and the effect of delayed diagnosis of cancer cases. CMAJ. 2006, 174:779-84. 10.1503/cmaj.050623

11. Rades D, Kronemann S, Meyners T et.al: Comparison of four cisplatin-based radiochemotherapy regimens for nonmetastatic stage III/IV squamous cell carcinoma of the head and neck. Int J Radiat Oncol Biol Phys. 2011, 80:1037-44. 10.1016/j.ijrobp.2010.03.033

12. Bensadoun RJ, Magné N, Marcy PY, Demard F: Chemotherapy-and radiotherapy-induced mucositis in head and neck cancer patients: new trends in pathophysiology, prevention and treatment. Eur Arch Otorhinolaryngol. 2001, 258:481-7. 10.1007/s004050100368

13. Jensen AD, Krauss J, Weichert W.: Disease control and functional outcome in three modern combined organ preserving regimens for locally advanced squamous cell carcinoma of the head and neck (SCCHN). Rad Oncol. 2011, 6:122. 10.1186/1748-717X-6-122 